\title{
Techniques for the production of point-light and fully illuminated video displays from identical recordings
}

\author{
SHARON M. THOMAS and TIMOTHY R. JORDAN \\ University of Nottingham, University Park, England
}

\begin{abstract}
Illumination of only a few key points on a moving human body or face is enough to convey a compelling perception of human motion. A full understanding of the perception of biological motion from point-light displays requires accurate comparison with the perception of motion in normal, fully illuminated versions of the same images. Traditionally, these two types of stimuli (point-light and fully illuminated) have been filmed separately, allowing the introduction of uncontrolled variation across recordings. This is undesirable for accurate comparison of perceptual performance across the two types of display. This article describes simple techniques, using proprietary software, that allow production of point-light and fully illuminated video displays from identical recordings. These techniques are potentially useful for many studies of motion perception, by permitting precise comparison of perceptual performances across point-light displays and their fully illuminated counterparts with accuracy and comparative ease.
\end{abstract}

A great deal of research in psychology has focused on the perception of motion. The visual perception of human motion is a valuable ability, since it provides essential cues for environmental interaction. One major phenomenon of the human perceptual system, first demonstrated by Johansson (1973), is the ability to perceive biological motion. In demonstrations of biological motion, a moving object (e.g., a human body) is represented by only a few bright spots describing the motion of the main joints. When the body is static, the perceiver's impression is one of a meaningless, random array of light points. However, when the body starts to move, seeing the dynamic light points is sufficient to convey a striking impression of a person performing specific activities (e.g., walking, running, jumping, lifting weights; Bingham, 1987; Johansson, 1973; Runeson \& Frykholm, 1981). Indeed, Johansson found that only 5-10 points give the visual system highly efficient information about human motion. Since then, many researchers have used Johansson's technique, and the information from such moving point-light displays has been shown to be sufficient for perceivers to determine the sex of the point-lit actor (Kozlowski \& Cutting, 1977; Mather \& Murdoch, 1994), to recognize the identity of the actor (Cutting \& Kozlowski, 1977; Stevenage, Nixon, \& Vince, 1999), or to decide which emotion is being bodily portrayed (Dittrich, Troscianko, Lea, \& Morgan, 1996).

These demonstrations indicate that the technique of point-light presentation is a highly useful tool in the study

Correspondence concerning this article should be addressed to $\mathrm{S}$. M. Thomas, School of Psychology, University Park, Nottingham NG7 2RD, England (e-mail: smt@ psyc.nott.ac.uk). of human perception of biological motion. Specifically, reduction of the complexity of perceptual input enables the essential parameters of human motion perception to be identified. Moreover, the preservation of a rich visual experience in a highly simplified representation has attractive implications for technological communication, as well as for computational modeling.

Recently, the point-light technique has also been applied to studies of moving human faces. Expression, age, and gender can be identified from moving light points attached to the face of an actor (Bassili, 1978; Berry, 1990), and the technique has also been used to great effect in investigations of audiovisual speech perception (e.g., Johnson, Rosenblum, \& Saldaña, 1994; Jordan, McCotter, \& Thomas, 2000; Rosenblum, Johnson, \& Saldaña, 1996; Rosenblum \& Saldaña, 1996). With fully illuminated talking faces, the facial movement accompanying speech production has been distinguished as a particularly powerful component of human communication because of its influence on auditory speech perception. Specifically, seeing the facial movements of the talker often helps people hear speech more clearly, in quiet and in noise (e.g., Erber, 1969; Sumby \& Pollack, 1954; Summerfield, 1979). Moreover, facial speech movement can alter the sound that we hear, as is demonstrated by the McGurk effect (e.g., McGurk \& MacDonald, 1976), where different auditory and visual inputs combine to form a new percept that was not presented in either modality alone. This influence of facial movement on speech perception has proven remarkably resistant to visual manipulations of the talking face, including changes in viewing angle, viewing distance, facial orientation, and facial color (Jordan \& Bevan, 1997; Jordan et al., 2000; Jordan \& Sergeant, 1998, 2000; Jordan \& Thomas, in press). 
When applied to talking faces, a static configuration of light points cannot be identified as a face. Nevertheless, when moving, these point light displays are recognized as unimodal visual speech and exert powerful influences on congruent (presented in noise) and incongruent auditory speech (Johnson et al., 1994; Rosenblum et al., 1996; Rosenblum \& Saldaña, 1996). These findings support the conclusion that pure dynamic information, as distinguished from other, pictorial visual information (color, shading, shapes, form), is important in audiovisual speech perception and recognition.

However, this research does have some fundamental problems to resolve. For example, from the studies reported thus far (e.g., Rosenblum et al., 1996; Rosenblum \& Saldaña, 1998), point-light faces do not show the same degree of visual influence on auditory speech perception as do fully illuminated faces (see also Dittrich et al., 1996). It could be argued that point-light faces were less influential because the arrangement of points in the displays used did not capture all aspects of kinematic visual speech information present in a fully illuminated face. Indeed, the optimal point-light configuration for visual and audiovisual speech perception is under investigation (e.g., Rosenblum et al., 1996; Rosenblum \& Saldaña, 1998). More fundamental problems, however, concern the techniques widely used to compare perceptual performance across point-light and fully illuminated displays. Of greatest concern is the fact that point-light and fully illuminated sequences have traditionally been filmed on separate occasions. As we will describe, this separate filming precludes precise comparison of perceptual performance across point-light and fully illuminated displays.

\section{Techniques for Producing Point-Light Displays}

A potentially attractive method for investigating human biological motion perception is to use artificially generated computer displays of biological motion (e.g., Ahlström, Blake, \& Ahlström, 1997; Bülthoff, Bülthoff, \& Sinha, 1998; Mather \& Murdoch, 1994; Neri, Morrone, \& Burr, 1998; Shiffrar, Lichtey, \& Chatterjee, 1997). Although these displays produce compelling impressions of moving objects and are potentially less cumbersome to produce than live recordings, they do not capture the precise nature or fluidity of real human motion. For ecological reasons, it is preferable to use displays of pointlight motion recorded from real moving objects. However, point-light displays of real moving human bodies and faces are still produced by using much the same techniques as those employed by Johansson (1973). In his first series of experiments on biological motion, small flashlight bulbs were wire-attached to a transformer, fitted into black funnel-shaped holders, and attached to the main joints of an actor dressed in tight-fitting dark clothing. The actor was filmed in a dark room so that only the lights could be seen. Johansson, noting that this technique was inconvenient and clumsy, reported a further technique whereby small patches of retroreflective tape were attached to the joints of the actor. The moving actor was then filmed under searchlights mounted close to the lens of a TV camera. The resulting videotape was then displayed on a TV monitor, the contrast of which was maximized. The patches of reflective tape therefore appeared as bright spots with sharp contours against a totally dark background (see also Dittrich et al., 1996). A similar experimental method is used for investigations of facial movement. Rosenblum and Saldaña (1996) filmed an actor whose skin was blackened with theatrical make-up. The actor's teeth were blackened with theatrical toothblack, and his tongue was darkened with food coloring. Twenty-eight 3-mm dots made from retroreflective tape were attached to the actor's blacked-out skin, using medical cement, and to the teeth and tongue, using a dental adhesive. The talking actor was then videotaped in low illumination so that only the reflective points were visible. The resultant edited video clips were presented to experimental participants on a monitor, appropriately adjusted for maximum contrast of dark background and white dots.

These studies have used essentially identical techniques for the production of point-light displays. As we have already indicated, however, there is a potentially serious problem with using these techniques. Specifically, the fully illuminated images are necessarily filmed separately from point-lit images. Consequently, it is essentially impossible to match two articulations precisely, even if they represent the same nominal stimulus spoken by the same individual. Variations may occur in the position of stimulus elements (e.g., leg position, head position, mouth position, etc.). Variations in lighting levels and the temporal nature of the action may also occur, and even if these variations are minute, the acuity of the human perceptual system would mean that these are detectable. To illustrate one aspect of this variation, we measured a number of aspects of a single talking face across six instances of uttering the same syllable. The talker was instructed to articulate precisely and identically over all utterances. Figure 1 illustrates the variation in lip separation across six instances of the same utterance (the syllable /ba/), three instances under point-light conditions and three instances under fully illuminated conditions. The extent of lip separation has concomitant effects on other facial dimensions, such as jaw and chin position, cheek bunching, and so forth.

As is evident from Figure 1, no two utterances have the same dimensions within conditions. Moreover, the extent of motion differs considerably between point-light and fully illuminated conditions, possibly owing to reluctance on the part of the talker to make gross movements with dots applied to the face. Moreover, the length of each utterance varied across instances, owing to differences in the time taken to reach maximal mouth opening (pointlight, $346 \pm 46 \mathrm{msec}$; fully illuminated, $307 \pm 61 \mathrm{msec}$ ) and the time to closure thereafter (point-light, $386 \pm$ $61 \mathrm{msec}$; fully illuminated, $453 \pm 61 \mathrm{msec}$ ). Throughout all these instances, the talker made an effort to articulate the syllable precisely and identically, but variation ap- 


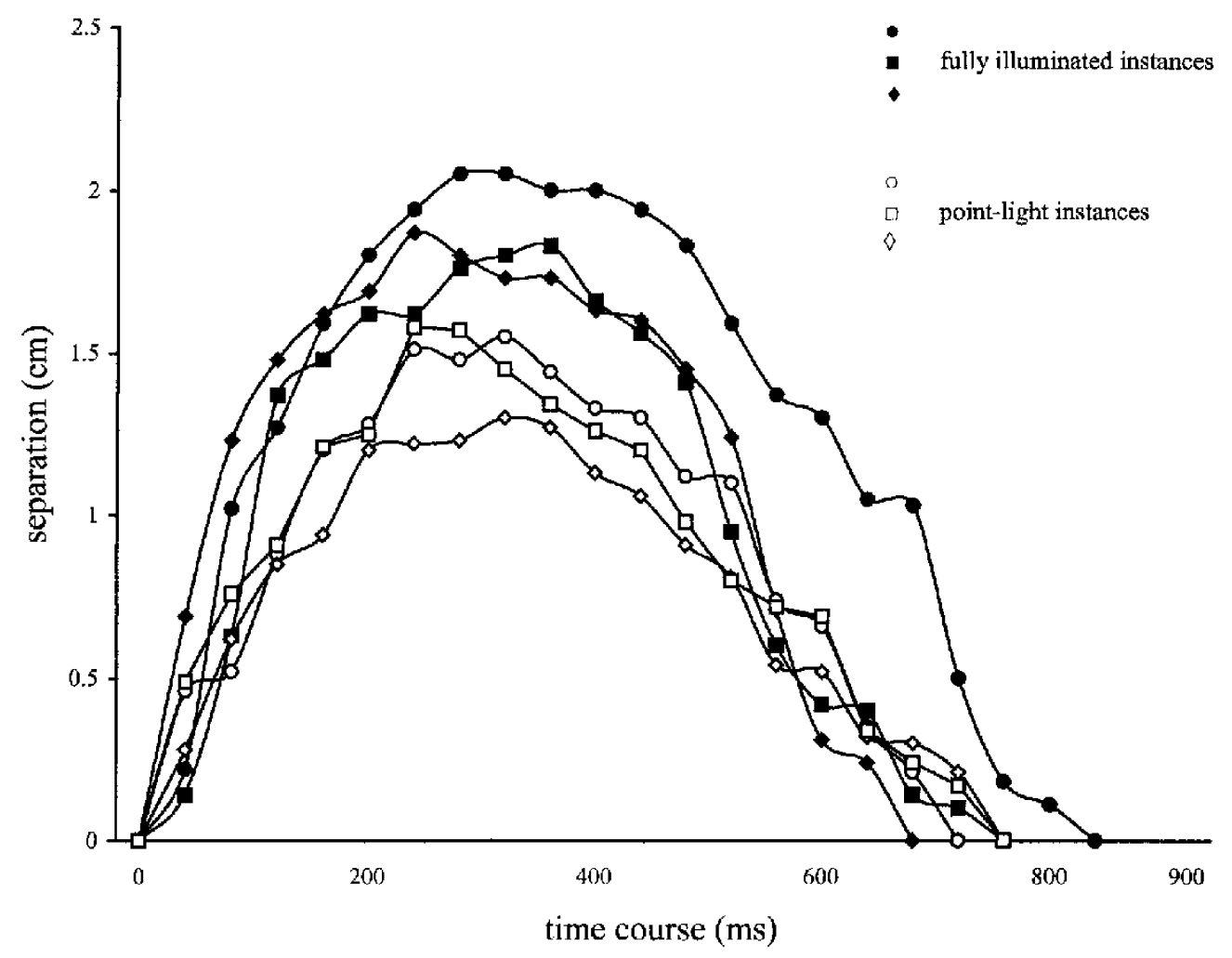

Figure 1. Variation in lip separation (in centimeters) over six separate instances of the utterance /ba/, three instances under fully illuminated conditions and three instances under point-light conditions.

pears to have been unavoidable. Even more variation may occur with larger body movements, such as walking and running (e.g., Yamada, 1995), which involve many more moving elements than those involved in speaking. When comparisons are being made across point-light and fully illuminated displays, therefore, natural and unavoidable variation may contaminate results. Ideally, for comparisons to be valid, the same stimulus instance should be used for point-light and fully illuminated displays. Recently, we have developed a simple technique for achieving this. Two simple modifications of video displays are described that permit precise and easy construction of exactly matched point-light and fully illuminated stimuli. Although the techniques are described specifically in terms of the production of talking-head video sequences, they are equally applicable to the production of any moving object displays.

\section{Stimulus Preparation}

Twenty-eight 3-mm dots were affixed to the face and articulators of a female actor, in the configuration described by Rosenblum and Saldaña, 1996; see Figure 2A). ${ }^{1}$ The dots were punched from a nonglossy white card and affixed to the face with spirit gum and to the lips and tongue with dental fixative. The technique we are about to describe eliminates the necessity to black out the face, oral cavity, or tongue of the person being recorded.

\section{Filming Set-Up}

A digital camera (Sony, DSR-200AP) was positioned on a tripod directly in front of and $2 \mathrm{~m}$ away from an actor. The camera was connected to a television monitor, providing an on-line view of the talking face throughout filming. Lighting was provided by three fluorescent lamps positioned above and to the front of the actor at $45^{\circ}$ (left and right). There were also two ultraviolet lights positioned at $45^{\circ}$ (left and right) close to the front of the actor, which served to enhance the whiteness of the dots on the face. The dimensions of the actor's head were noted, and the zoom function of the camera was adjusted so that the head proportions on the monitor were accurate. Simultaneous 16-bit audio recordings were made via a clip microphone (Sony Electret Condenser ECM-44B) with input to the camera.

\section{Editing}

When filming was complete, the digital videotape was played back, using a digital videocassette recorder linked to a Power Mac via Firewire. Video clips of all the stimulus items were then captured from the original digital footage, using Radius edit DV capture software. Each of these clips was then modified in two different ways to produce point-light displays (everything but the dots removed) and fully illuminated displays (dots removed from the talking head). 


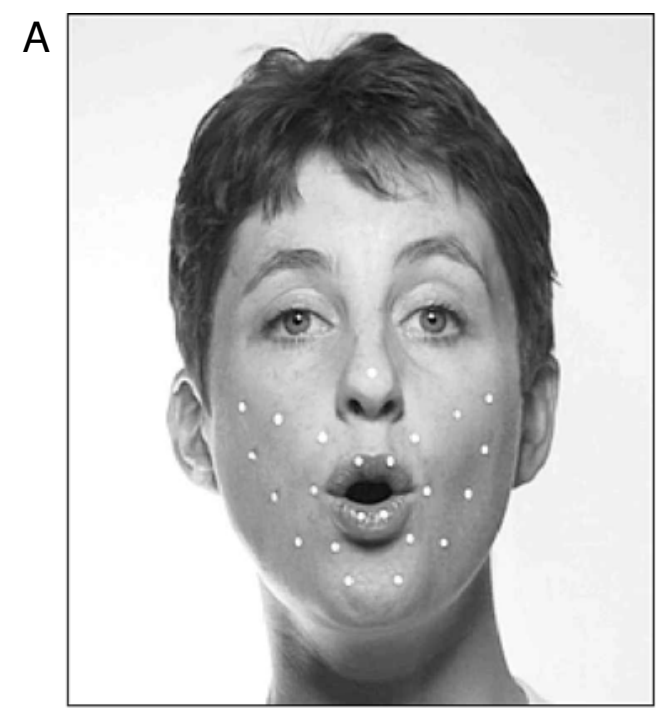

\section{B}

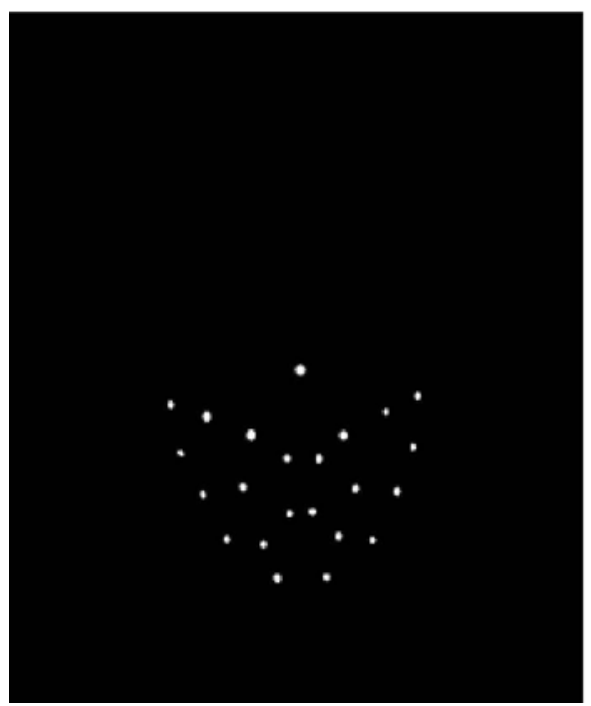

C

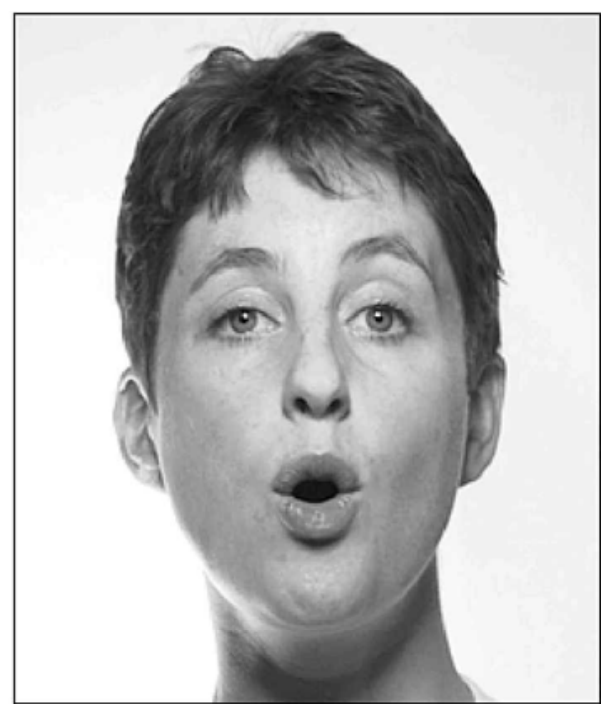

Figure 2. (A) Frame taken from video footage showing twenty-eight 3-mm reflective dots affixed to the face and articulators of a female actor, in the configuration described by Rosenblum and Saldaña (1996; five dots, placed on the front teeth and tongue tip, are not visible in this image). The original images were in color. (B) Frame taken from edited video footage with all unwanted colors removed, so that only white elements remain. (C) Frame taken from edited video footage with reflective dots removed by motion-tracking and cloning techniques. The originalimages were in color.

\section{Point-Light Displays}

The captured stimulus clips were modified using Adobe After Effects video processing software so that only the luminous points were visible. This was achieved by use of color-changing filters that allowed the extraction of all unwanted colors within the video clip, leaving only the white pixels. Specifically, an unwanted color in the image (e.g., a flesh tone) was selected by using the color selection eye-dropper tool, which enabled precise pixel- accurate choice of color. The hue, saturation, and lightness of the selected color were then reduced, resulting in the color's turning to black. The application of the color filters is automatic once selected - that is, there is no need for frame-by-frame editing, since the process is applied to the entire clip. This process was repeated with all unwanted colors, so that only white elements remained and the final result was a pattern of moving dots on a black background (see Figure 2B). Additional necessary mod- 
ifications (e.g., eliminating the whites of the eyes) were carried out by using the Commotion editing software cloning function (see below).

\section{Fully Illuminated Displays}

The point-light dots were removed from the face, using the motion-tracking and clone functions of Commotion video postproduction software. The motiontracking function enabled the precise tracking of each dot on the face from frame to frame, and the clone function enabled replacement of the dots along the motion path with pixels cloned from the immediate surrounding regions. Specifically, a single dot was motion-tracked, so that its motion in terms of $x / y$-coordinates was precisely mapped from frame to frame. The tracking was based on the luminance values within the image, using the high degree of contrast between the luminous dots and the surrounding facial texture. The result was displayed as a vector path. By using the clone function, pixels were then copied from reference points surrounding the white dot and applied to the dot itself, essentially "painting out" the dot with surrounding flesh tones. The precise clone reference points and brush strokes used were determined on the first frame of the sequence and were recorded by the software. The reference point and brush stroke information was then automatically applied to every point on the dot's motion path. The application of the brush strokes is automatic once selected-that is, there is no need for frame-by-frame editing, since the process is applied to the entire clip. This ensured a smooth elimination of the dot from the entire clip. This procedure was applied to every dot on the talking-head clip, and the final result was a video clip of a normal talking face.

The combined use of motion tracking and recorded brush strokes is important for two main reasons. First, if the luminous dots are simply painted out on every individual frame, the task is not only very time consuming, but more important, the result is an irregular patch of pixels moving throughout the clip, because the precise placement of identical pixels over each dot is exceedingly difficult. The repeated placement of recorded brush strokes on every frame provides this precision, and because of the motion tracking, the strokes follow the smooth vectordefined path of the moving dot. The video images created by using the above sequence of techniques were of a normal, fully illuminated, digital quality talking face (see Figure 2C). The images were assessed by independent observers under testing conditions as being indistinguishable in terms of skin tone and texture from normally filmed, unedited talking faces - that is, the observers could not tell where the dots had been before editing. The entire process of creating these sequences (point-light and fully illuminated) takes, on average, 10 min of time for a 4-sec clip (the standard clip length for spoken word stimuli). Applying the effects to longer clips (e.g., spoken sentences) naturally takes more time, but the processing is mostly automated and, relative to alternative methods, efficient and simple. Although all our production was carried out on a Power Mac, the software applications used (Adobe After Effects, Commotion) are available for both Mac and Windows platforms.

Stimulus items constructed by using the above techniques have been successfully used in several of our experiments that investigated the perception of audiovisual speech from point-light and fully illuminated displays. We will specifically describe one study in which perception of visual and audiovisual speech from point-light and fully illuminated faces was compared. The study was conducted to assess the effects on audiovisual speech perception of changing the viewing angle from which faces are observed. Point-light and fully illuminated versions of talking faces filmed from three angles (fullface, three-quarters, profile) were generated by applying the techniques outlined in this paper. We produced pointlight and fully illuminated versions of several congruent audiovisual words and incongruent audiovisual pairings known to produce strong McGurk effects (e.g., /bay/ /gay/ = perception/day/). Clips were also included where no facial movements occurred (auditory signal only) and where no auditory signal occurred (visual signal only). Analysis of the data from the visual only, congruent, and incongruent audiovisual conditions revealed main effects of display type, reflecting the fact that point-light stimuli were less effective than fully illuminated stimuli, although this did not interact with viewing angle. Results with our technique have generally indicated a high level of performance with the point-light stimulus items, although these performance levels are rarely as good as those observed with fully illuminated stimuli. This finding is in keeping with previous studies conducted using point-lightdisplays of talking faces and, indeed, is complementary to the finding of Massaro and colleagues (e.g., Cohen, Walker, \& Massaro, 1996), who showed that the influence of visible speech is much weaker with point-light displays created from an animated (computer-generated) talking head than with the same talking head. Interestingly, when the data from this study were compared with data from previous point-light studies that used separately recorded point-light and fully illuminated sequences (e.g., Jordan et al., 2000), the decrement in performance with point-light displays of audiovisual speech was larger, as compared with that observed in earlier studies. Although it would be premature to suggest that this finding is conclusive, the fact that differences are observed with different display production techniques emphasizes the need to exercise maximum control in experiments by using the same recorded footage to produce point-light and fully illuminated displays. We are, therefore, in a position to confidently conclude that the kinematic information provided by point-light displays is sufficient for accessing the human audiovisual speech recognition system but that there is additional information contained in fully illuminated faces that is used by this system. This confidence has been considerably strengthened by the knowledge that the two displays, point-light and fully illuminated, were precisely matched. This assurance, as well as the accuracy and rel- 
ative ease with which the talking-face images were produced, is transferable to any type of point-light display.

\section{Conclusion}

The point-light technique has proven to be a highly useful tool for reducing the complexity of perceptual input and thereby investigating the essential nature of internal representations underlying human dynamic threedimensional object recognition. Although a number of techniques are available that allow the investigator to produce point-light displays of moving objects, these methods do not allow precise matching with fully illuminated controls. Specifically, the fully illuminated displays must be filmed separately from the point-light displays, which allows uncontrolled variation across displays. The present article shows how point-light and fully illuminated displays can be produced from a single, identical recording, thereby enabling accurate comparison of human perceptual performance across displays. Although the examples used in this article were specific to moving, talking facial images, the techniques can work equally well on other moving objects.

\section{REFERENCES}

Ahlström, V., Blake, R, \& Ahlström, U. (1997). Perception of biological motion. Perception, 26, 1539-1548.

BAssiLI, J. N. (1978). Facial motion in the perception of faces and of emotional expression. Journal of Experimental Psychology: Human Perception \& Performance, 4, 373-379.

BerRy, D. S. (1990). What can a moving face tell us? Journal of Personality \& Social Psychology, 58, 1004-1014.

Bingham, G. P. (1987). Scaling and kinematic form: Further investigations on the visual perception of lifted weight. Journal of Experimental Psychology: Human Perception \& Performance, 13, 155-177.

Bülthoff, I., Bülthoff, H., \& Sinha, P. (1998). Top-down influences on stereoscopic depth-perception. Nature Neuroscience, 1, 254-257.

Cohen, M. M., Walker, R. L., \& Massaro, D. W. (1996). Perception of synthetic visual speech. In D. G. Stork \& M. E. Hennecke (Eds.), Speechreading by humans and machines (pp. 153-168). New York: Springer-Verlag.

Cutting, J. E., \& Kozlowski, L. T. (1977). Recognizing friends by their walk: Gait perception without familiarity cues. Bulletin of the Psychonomic Society, 9, 353-356.

Dittrich, W. H., Troscianko, T., Lea, S. E. G., \& Morgan, D. (1996). Perception of emotion from dynamic point-light displays represented in dance. Perception, 25, 727-738.

ERBER, N. (1969). Interaction of audition and vision in the recognition of oral speech stimuli. Journal of Speech \& Hearing Research, 12, 423-425.

Johansson, G. (1973). Visual perception of biological motion and a model for its analysis. Perception \& Psychophysics, 14, 201-211.

Johnson, J. A., Rosenblum, L. D., \& Salda Ña, H. M. (1994, April). The contribution of a reduced visual image to speech perception in noise. Poster presented at the 127th Meeting of the Acoustical Society of America, Cambridge, MA. (Abstract in Journal of the Acoustical Society of America, 95, 3009.)

JoRDAN, T. R., \& BEVAN, K. (1997). Seeing and hearing rotated faces: Influences of facial orientation on visual and audiovisual speech rec- ognition. Journal of Experimental Psychology: Human Perception \& Performance, 23, 388-403.

Jordan, T. R., McCotter, M. V., \& Thomas, S. M. (2000). Visual and audiovisual speech perception with color and gray-scale facial images. Perception \& Psychophysics, 62, 1394-1404.

Jordan, T. R., \& Sergeant, P. C. (1998). Effects of facial image size on visual and audio visual speech recognition. In R. Campbell, B. Dodd, \& D. Burnham (Eds.), Hearing byeye: Pt. 2. The psychologyof speechreading and audiovisual speech (pp. 155-176). London: Taylor \& Francis.

Jordan, T. R., \& SERgeant, P. C. (2000). Effects of distance on visual and audiovisual speech recognition. Language \& Speech, 43, 107-124. JoRDAN, T. R., \& THOMAS, S. M. (in press). Effects of horizontal viewing angle on visual and audiovisual speech recognition. Journal of Experimental Psychology: Human Perception \& Performance.

Kozlowski, L. T., \& CutTING, J. E. (1977). Recognizing the sex of a walker from a dynamic point-light display. Perception \& Psychophysics, 21, 575-580.

MATHER, G., \& MURDOCH, L. (1994). Gender discrimination in biological motion displays based on dynamic cues. Proceedings of the Royal Society of London: Series B, 258, 273-279.

McGURK, H., \& MACDonALD, J. (1976). Hearing lips and seeing voices. Nature, 264, 746-748.

Neri, P., Morrone, M. C., \& Burr, D. C. (1998). Seeing biological motion. Nature, 395, 894-896.

Rosenblum, L. D., Johnson, J., \& Saldaña, H. M. (1996). Visual kinematic information for embellishing speech in noise. Journal of Speech \& Hearing Research, 39, 1159-1170.

Rosenblum, L. D., \& SAldaña, H. M. (1996). An audiovisual test of kinematic primitives for visual speech perception. Journal of Experimental Psychology: Human Perception \& Performance, 22, 318-331.

Rosenblum, L. D., \& SaldaÑa, H. M. (1998). Time-varying information for visual speech perception. In R. Campbell, B. Dodd, \& D. Burnham (Eds.), Hearing by eye: Pt. 2: The psychology of speechreading and audiovisual speech (pp. 61-81). London: Taylor \& Francis.

Runeson, S., \& FrY KHOLM, G. (1981). Visual perception of lifted weight Journal of Experimental Psychology: Human Perception \& Performance, 7, 733-740

Shiffrar, M., Lichtey, L., \& ChatterJee, S. H. (1997). The perception of biological motion across apertures. Perception \& Psychophysics, 59, 51-59.

Stevenage, S. V., Nixon, M. S., \& Vince, K. (1999). Visual analysis of gait as a cue to identity. Applied Cognitive Psychology, 13, 513-526.

Sumby, W. H., \& Pollack, I. (1954). Visual contribution to speech intelligibility in noise. Journal of the Acoustical Society of America, 26, 212-215.

SuMMERFIELD, A. Q. (1979). Use of visual information in phonetic perception. Phonetica, 36, 314-331.

YAMADA, N. (1995). Nature of variability in rhythmical movement. Human Movement Science, 14, 371-384.

\section{NOTE}

1. We may also recommend an alternative method for dot placement on the skin of the actor. The dots may be painted directly on to the face of the actor, using luminous face paint. Filmed under ultraviolet light, these dots are very effective and stand out in sharp contrast to the skin and articulators of the actor. Very precise care must be taken, however, to ensure that the painted dots are identical. This can be achieved through the use of a stencil.

(Manuscript received March 29, 2000; revision accepted for publication December 13, 2000.) 